\title{
Bursitis trochanterica
}

\author{
Ist ein Gelenksersatz notwendig oder nicht? \\ Ein Fallstrick, der meist ältere Damen, aber auch junge Sportler betrifft
}

In dieser Rubrik präsentieren wir Ihnen interessante Fälle oder Fallstricke aus der Praxis. Wir laden Sie weiterhin recht herzlich ein, interessante und praxisrelevante Fälle zu präsentieren und ersuchen Sie um Übermittlung der dementsprechenden Manuskripte an: redaktion@sportmedizin.or.at Geben Sie Ihre Erfahrungen weiter und profitieren Sie von den Erfahrungen der Sport- und präventivmedizinischen Community!

Die Bursitis trochanterica ist ein Krankheitsbild, das ich während meiner Ausbildung nicht ein einziges Mal gesehen habe, aber im ärztlichen Alltag sehr häufig vorkommt.

Die Patienten kommen in die Ordination mit Hüftschmerzen und der Befürchtung, ein neues Gelenk zu benötigen. Meist sind es ältere Damen, seltener junge Sportler.

\section{Diagnose}

Bei der Untersuchung findet sich ein in allen Ebenen frei bewegliches Hüftgelenk, ein starker Druckschmerz über dem Trochanter major und Ausstrahlungsschmerzen an der Außenseite des betroffenen Beines.

Die Diagnose ist meist einfach und eindeutig - Bursitis trochanterica.

Als Ursache für die Bursitis findet sich anamnestisch oft eine längere ungewohnte Belastung, wie beispielsweise der Beginn eines Nordic Walking Trainings, oder Gangstörungen nach Hüft oder Knieoperationen. Bei jungen Sportlern ist meist eine Überbelastung beim Sport die Ursache.

Bei der Untersuchung des Patienten stellt man als Begleitsymptome oft eine Beinlängendifferenz oder ein Hinken fest.

\section{Abb. 1: Ein schmerzfreier Hüftschwung...}

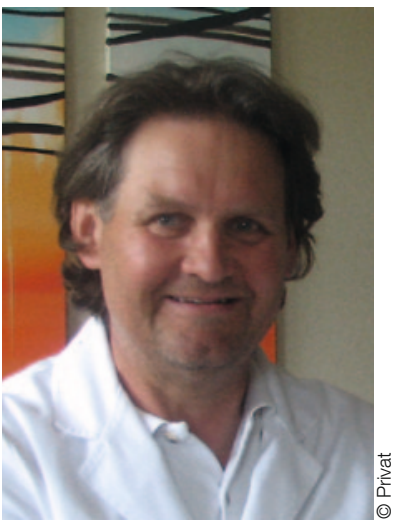

Martin Nehrer

\section{Therapie}

Die Therapie besteht in der Verordnung von NSAR, physikalischer Therapie mit Ultraschall, Stereo-Dynator, (cave Implantate!) Fango und Moorpackungen.

Am besten bewährt hat sich allerdings eine Infiltration in und an die Bursa trochanterica mit Xyloneural und einem Corticosteroid.

Die Patienten sind daraufhin rasch beschwerdefrei und sehr dankbar, weil sie einerseits keinen Schaden des Hüftgelenkes haben und nicht operiert werden müssen, andererseits wieder Alltagsbelastungen aushalten und beispielsweise wieder auf der betroffenen Seite schlafen können.

Zum Abschluss werden dem Patienten noch Dehn und Kräftigungsübungen der Hüft- und Beinmuskulatur empfohlen und auch demonstriert, um ein Wiederauftreten der Symptomatik zu verhindern.

Es lohnt sich also beim Hüftschmerz, an dieses Krankheitsbild zu denken. Es kommt öfter vor als man glaubt.

Korrespondenz:

Dr. Martin Nehrer

Arzt- für Allgemeinmedizin, Sportarzt

Arzt der Landesstelle Burgenland der SVA der Gewerblichen

Wirtschaft

F. Lisztgasse 1

7000 Eisenstadt

E-Mail: doc.nehrer@bkf.at

Tel.: 02682/651 21 